$\xi=-1$

\title{
Binary Plane Technique Based Color Quantization for Content Based Image Retrieval
}

\author{
T Esther Ratna ${ }^{1}$, N Subash Chandra ${ }^{2}$ \\ ${ }^{I}$ Research Scholar, Department of Computer Science, Rayalaseema University, Kurnool, Andhra Pradesh, India. \\ ${ }^{2}$ Professor, Department of CSE, CVR College of Engineering, Hyderabad, Telangana, India. \\ *Corresponding author E-mail: esther.subodh@gmail.com
}

\begin{abstract}
Extracting accurate informative file from a high volume of graphic files is a challenging task. This paper focus on presenting a new color indexing approach using the histogram features. Two histogram features like maximum color histogram and minimum color histogram are computed and are vector quantized to constitute a feature vector. Bit plane technique is used to map these features based upon it value at the respective position. The ultimate goal of any retrieval method is to attain higher precision within a short span of time that could be achieved if the data is in compressed to accomplish this the image is compressed using binary plane technique. The result analysis depicts the performance of the proposed approach under lossy and lossless modes and found that when operated in lossy it attain effective precision rate in a speculated amount of time.
\end{abstract}

Keywords: Binary Plane Technique, Image Retrieval, Color Quantization and Histogram Features.

\section{Introduction}

Retrieving the data based on text keywords as query is what it is observed in many of the search engines and digital libraries but it involves many tasks in retrieving the image data. Since text is the man made creation but images is that one has seen from his early ages and becomes more difficult to characterize each image to train a machine to retrieve its relevant image samples, this makes the research of image retrieval more unique and demand in research. No universally acceptable algorithm is proposed for characterizing human vision hence the research and an effort to explore new methods is a continuous process.

Content based image retrieval (CBIR) is an approach to extract the relevant images from the digital library using visual features like color, shape and texture. The goal of image retrieval system is to retrieve the images with higher precision in a lower time interval so that any human intervention is limited or avoidable. The major advantages of CBIR approach are the possibility of an automatic retrieval, instead of the traditional approach (keyword-based) approach, which is usually very laborious and time-consuming as compare to previous annotation of database images. The CBIR approach had been used for several applications such as fingerprint identification system, biodiversity information, digital libraries, crime prevention, medicine detail system, historical research, among others [1].

The digital data is projected onto spatial domain with high volume of graphical content that requires large amount of space for storage and require larger bandwidth for transmission. The need for accessing such content is increasing day by day, in order to meet these requirements the content should be compressed and efficient retrieval techniques has to be employed for extracting the most relevant information data. The methods require having a high precision with high speed of processing. This can be achieved jointly with two different mechanisms in image processing (retrieval with compression). Nevertheless, it can be observed that a best image compression has to employed that even retains the maximum features of image data for retrieval [2].

Most of the standard compression techniques like JPEG does not consider the retrieval process [3], however there are some methods like vector Quantization (VQ) which involve this despite of suitable power however they are observed to be slow for compression [4]. In simpler terms, it can be termed as the problem of showing an image with high efficiency in binary form. Binary plane technique [5] [6] is one of the solutions that can be incorporated with color feature based CBIR systems to attain higher precision at faster rates.

This paper is organized as follows; section 1 presents the need and necessity of the work and the concepts related to the work. Section 2 presents the earlier research work conducted for retrieval systems; section 3 presents the generalized structure of the work explaining each step in detail. Section 4 presents the experimental results and its performance analysis ending up with conclusions.

\section{Related Work}

In this paper the research articles related to color features and retrieval systems for compressed data which are proposed in later 2000 are focused

In [5] S.Mahaboob Basha et.al, presented binary plane technique (BPT) that takes the advantage of repetitive values in the successive positions.

In [6] Subhash et.al, presented a binary difference with Huffman coding (BDH) that shows the effect of using difference coding with Basha's BPT approach. 
In [7] Deng et.al, presented a region based color descriptor that is indexed in $3 \mathrm{D}$ color space. This also includes the percentage of coverage with in the speculated region. This method proved to be better than traditional histogram approaches by providing dimensionality reduction of feature vector.

In [8] Jeong et.al, proposed Gaussian mixture vector quantization to extract the color histograms and showed better retrieval results than uniform quantization.

In [9] manjunath et. al, presented retrieval system using color and texture descriptors of MPEG-7 standard which attained satisfactory results for images and videos using histogram based descriptor, dominant color descriptor, spatial color descriptors which are well suited for browsing based applications.

In [10] Gahroudi et. al presented block truncating coding based image retrieval system with vector quantization. In this work they used Color histograms and block pattern histograms which are used to provide suitable information that increases the speed and efficiency of the system.

In [11] Sergyan, presented color histogram feature based image retrieval in which the statistical parameters like mean, standard deviation, skewness, energy of the histogram were considered as features and different distance transforms were used to find the similarity score. This approach is very fast, simpler to implement and efficient in retrieval.

In [12] Lu et.al , presented color image retrieval using color features and bit map which is used to represent the local characteristics of the image for increasing the accuracy of the system. It is also proved that the method consumes less memory for storing the features of the image.

In [13] poursistai et.al proposed effective image retrieval system in JPEG compressed domain, the features are extracted for the compressed images where the vector quantization is employed to minimize the dimensionality of the feature vector. The method proved to be more effective than other color feature methods and with higher precision values.

In [14] Guo et.al presented retrieval system with color quantization with block truncating coding and vector quantization. The work consists of two new color features named as color histogram feature, bin pattern histogram feature which are directed to vector quantization to form color quantizer and indexed bit map image. This word is not only superior to traditional BTC (block truncating coding) approach but also with regards to the other retrieval systems in compressed domain.

In [15] Ratna et.al presented, the process of bit map generation using BTC and EDBTC (Error diffusion block truncating coding) and their application for image retrieval.

\section{Proposed Frame Work}

Figure $1 \& 2$ depicted below represents the process of feature vector calculation and similarity measurement for the retrieval process.

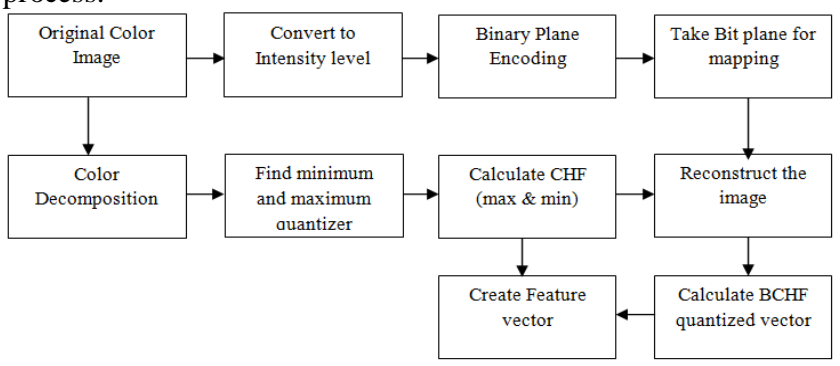

Fig. 1: Block diagram to extract the feature for retrieval

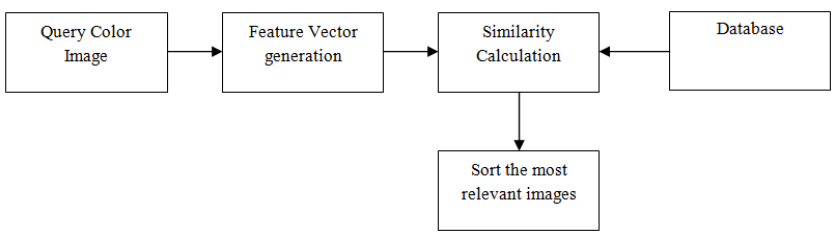

Fig. 2: Block diagram for similarity measurement

\subsection{Pseudo Code for Retrieval Process}

- The images are collected from standard databases which are resized to $256 \times 256$ and stored forming a database for analysis.

- The image is converted to gray scale which is then followed to binary plane coding; the outcome of this coding process contains data plane, biplane and indexing matrix

- The original color image is decomposed and the color quantizers are computed by looking for the minimum and maximum of all image pixels in each image.

- The probability distributions of quantized vectors are calculated, thus forming minimum and maximum color histogram feature (CHF) vector.

- $\quad$ The obtained bit plane from bit plane coding is used to map the maximum and minimum color quantized values forming biplane based color histogram feature(BCHF)

- $\mathrm{CHF}_{\max }, \mathrm{CHF}_{\min }, \mathrm{BCHF}$ all put together form the feature vector for retrieval process.

\section{(a) Color decomposition}

Let us consider a color image $f(x, y)$ of size $\mathrm{MxN}$, then it can be mathematically represented as

$f(x, y)=\left\{f_{y}(x, y), f_{g}(x, y), f_{b}(x, y)\right\}$

Where $x=1,2 \ldots M$ and $y=1,2 . . N$

The inter-band average value can be computed as

$\bar{f}(x, y)=\frac{1}{a}\left(f_{Y}(x, y)+f_{g}(x, y)+f_{b}(x, y)\right)$

In the above equation $f_{r}(x, y)$, represents the red colored pixels in the image and similarly for green and blue respectively. The average all theses band constitute the gray scale image. Consider two quantizers for these color bands that were obtained by considering the maximum and minimum values of the bands. The color quantizers are mathematically represented as

$q_{\min }(x, y)=\min \left\{f_{f}(x, y), f_{g}(x, y), f_{b}(x, y)\right\}$

$q_{\max }(x, y)=\max \left\{f_{Y}(x, y), f_{g}(x, y), f_{b}(x, y)\right\}$

Thus obtained color quantizers along with the constructed bitmap are sent to the decoder through transmission channel. At the decoder, the maximum quantized values are place at the positions of ' 1 ' in the bitmap and minimum quantized are placed at the positions of ' 0 '. The decoder is very simpler to implement as it doesn't require any computation to reconstruct the image this makes it more attractive for real time applications.

\section{(b) Vector Quantization (VQ)}

In vector quantization the codebook is constructed by iteratively partitioning the source vector with the help of some statistical features that are able to produce code vectors with very little distortion.

Let $C=\left\{c_{1}, c_{2}, \ldots, c_{n}\right\}$ be the color code book that was generated using VQ method that consists $\mathrm{N}_{\mathrm{c}}$ code words however this VQ requires large number of images for training. The vector $c_{k}$ contains RGB color (or gray scale) information which is identical to the two color quantizers.

Given the color codebook $\mathrm{C}=\left\{\mathrm{c} 1, \mathrm{c} 2, \ldots, \mathrm{C}_{\mathrm{N}}\right\}$, the VQ indexes the EDBTC minimum and maximum quantizers using

$\bar{i}_{\min }\left(i_{s} j\right)=\min \left\|q_{\min }\left(i_{v} j\right), c_{k}\right\|_{2}^{2}$

$\bar{i}_{\max }\left(i_{s} j\right)=\max \left\|q_{\max }\left(i_{s} j\right), c_{k}\right\|_{2}^{2}$

The VQ reduces the bit required for storing the color minimum and maximum quantizers by performing the indexing process. 


\section{(c) Color histogram Features (CHF)}

The CHF comprises of two quantizers, $\mathrm{CHF}_{\min }$ and $\mathrm{CHF}_{\max }$ that are constructed from two color quantizers. These two quantizers capture the color content information for a given image. This information alias features gives the information about the pixel brightness and color distribution.

The $\mathrm{CHF}_{\text {min }}$ and $\mathrm{CHF}_{\text {max }}$ features can be computed using

$$
\begin{aligned}
& C H F_{\min }=\operatorname{Pr}\left\{\bar{t}_{\min }\left(i_{i} j\right)\right\} \\
& C H F_{\max }=\operatorname{Pr}\left\{\bar{\imath}_{\max }\left(i_{i} j\right)\right\}
\end{aligned}
$$

Where $i=1,2 . . M, j=1,2, \ldots N$

The $\mathrm{CHF}_{\text {min }}$ and $\mathrm{CHF}_{\text {max }}$ are the VQ-indexed histogram from the color minimum and maximum quantizers, respectively. It only calculates the occurrence of certain color code words appeared in an image.

\section{(d) Binary Plane Coding}

The Binary Plane Techniques are applied for color images as well as for gray scale images. In this technique the compressed file is categorized into two, a bit plane and a data table. The first consists of ' 1 ' and ' 0 's that represent whether the pixel is unique or repetitive. Secondly, the data table has only the unique pixel values, both these are merged into one file.

This method can be applied in both modes (i) Lossless and (ii) Lossy. The loss less compression technique is based on spatial domain of the image and they are very much suitable for the compression of medical images. The Lossy Binary Plane technique introduces little loss to achieve more compression rate. The principle of this method is to take the advantage of repetitive values that are appeared successively. In this method instead these repetitions only one value is considered. In brief, the binary plane approach has two planes and the bit planes consist of 0's and 1's.

The codes are as given below

Code 1 (one) is used to indicate that current pixel is different from previous pixel. In this case the current pixel is moved to the Data Table

Code 0 is used to indicate that the current pixel is exactly same as previous pixel. This eliminates the storage of current pixel. [5] [6] [16] [17].

\section{(e) Construction of Binary plane based color histogram feature}

This is the unique feature of the work which makes to retain higher precision value. This is formed by indexing the bit plane obtained from binary plane coding to two color quantized outputs. The new image is reconstructed as represented in equation (9) and its VQ code word is calculated using equation (10) which is used as feature vector forming $\mathrm{BCHF}$ as represented in equation (11)

$r\left(i_{i} j\right)= \begin{cases}c_{\max }\left(i_{s} j\right) & \text { if } b p\left(i_{v}, j\right)=1 \\ c_{\min }\left(i_{,} j\right) & \text { if } b p(i, j)=0\end{cases}$

$\bar{r}\left(i_{v} j\right)=\left\|r\left(i_{s} j\right), c_{k}\right\|_{2}^{2}$

$B C H F=\operatorname{Pr}\{r i(i, j)\}$

\section{(f) Similarity Calculation}

The similarity for the retrieving the relevant data is measured using the following equation

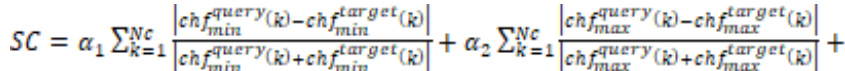

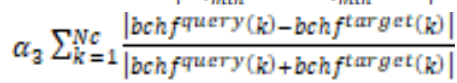

Where $\mathrm{Nc}$ is the color histogram bins ins and $\alpha_{1,23}$ are constant representing the contribution of $\mathrm{CHF}$ and $\mathrm{BCHF}$

\section{Experimental Results}

The experiments were conducted using COREL Image database for image retrieval available at [18], the Corel dataset consists of 10,908 different images with the size of $256 \times 384$ for each image. These groups of datasets are Buses, Mountains, Beach, Elephants, Food, Flowers Africa, Horses, Dinosaurs and Buildings. The results were reported using these groups because most of the remarkable researches [19] [20].

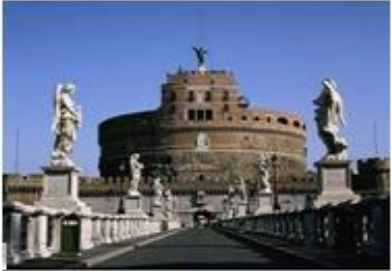

(a)

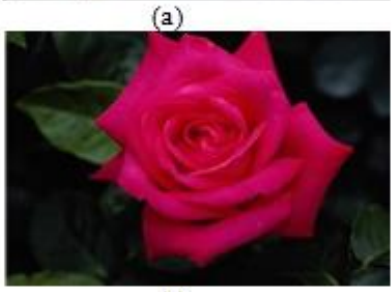

(c)

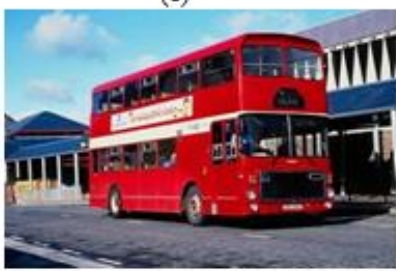

(e)

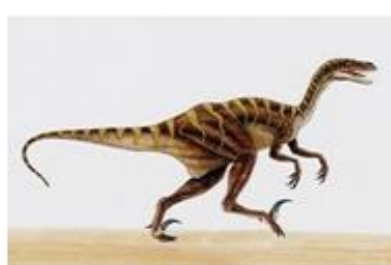

(b)

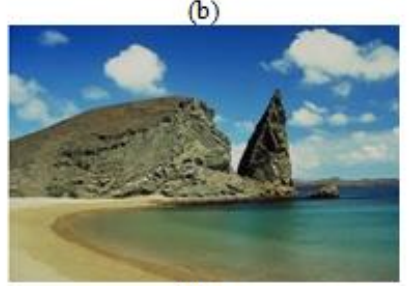

(d)

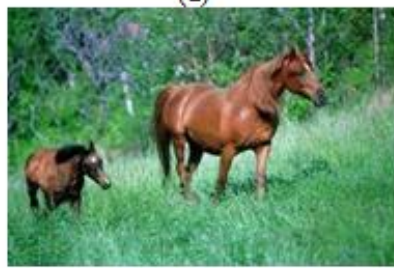

(f)
Fig. 3: Images used for experimental analysis (a) Buildings (b) Dinosoroaus (c) Flower (d) Sea shore (e) Buses (f) Horses

The images used in the experiment are resized to $256 \times 256$ for computation purpose; the performance of the proposed approach is calculated in terms of precision and recall [21]. The performance is evaluated using Matlab 2016a version tool with 4GB RAM.

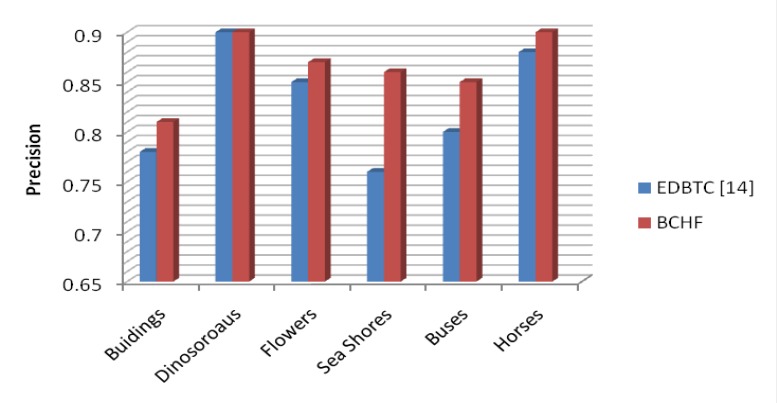

Fig. 4: Performance of the proposed approach with precision values

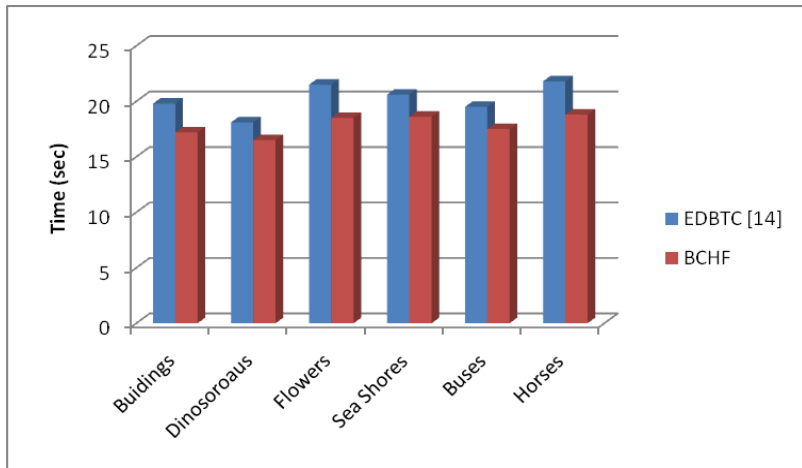

Fig. 5: Performance of the proposed approach in terms of elapsed time

From the analysis it is observed that the proposed approach attaining high precision of about $20 \%$ when compared against the meth- 
od proposed in [14], however it consumes very less time for re- [18] trieval which is around $25 \sim 30 \%$ on average for different images. Thus, achieving the main objective of the work in retrieving relevant images with in short span of time.

\section{Conclusion}

A new binary plane based color histogram feature is proposed for CBIR system. From the experimental analysis it is observed that the proposed approach could achieve the objective of the work and able to perform better than the traditional approach. This method can be further extended with analyzing the precision, recall and processing time with lossy mode of binary plane operation under different thresholds.

\section{References}

[1] A.W.M. Smeulders, M. Worring, S. Santini, A. Gupta, R. Jain, "Content- Based Image Retrieval at the End of the Early Years," IEEE Transactions on Pattern Analysis and Machine Intelligence, vol. 22, no. 12, pp. 1349-1380, 2000

[2] S. Pachanathan, "'Compressed or Progressive Image Search", Image Database: Search and Retrieval of Digital Imagery, Wiley \& Sons, pp.465-495, 2002.

[3] M. Shenier and M. Abdel-Mottaleb, "Exploiting the JPEG Compression Scheme for Image Retrieval", IEEE Transactions on Pattern Analysis and Machine Intelligence, Vol. 18, No. 8, pp.849-853, 1996.

[4] F. Idris and S. Panchanathan, "Image indexing using vector quantization", Proc. SPIE: Storage and Retrieval for Image and Video Databases III, pp. 373-380, 1995

[5] S.Mahaboob Basha and Dr. B. Sathyanarayana, "Image Compression Using Binary Plane Technique," IEEE, vol. 1, no. 1, pp. 4-65, 1996

[6] N. Subhash Chandra et al., "Loss less compression of images using binary plane, difference and huffman coding (BDH technique)," Journal of Theoretical and Applied Information Technology, vol. 3, no. 1, pp. 3-56, 2008

[7] Y. Deng, B. S. Manjunath, C. Kenney, M. S. Moore, and H. Shin, "An Efficient Color Representation for Image Retrieval," IEEE Trans. Image Processing, 10(1):140-147, 2001.

[8] S. Jeong, C. S. Won, and R.M. Gray, "Image retrieval using color histograms generated by Gauss mixture vector quantization," Computer Vision and Image Understanding, 9(1-3):44-66, 2004.

[9] B. S. Manjunath, J.-R. Ohm, V. V. Vasudevan, and A.Yamada, "Color and Texture Descriptors," IEEE Trans. Circuits and Systems for Video Technology, 11(6):703-715, 2001.

[10] M. R. Gahroudi and M. R. Sarshar, "Image retrieval based on texture and color method in BTC-VQ compressed domain," in Proc. Int. Symp. Signal Process. Appl., Feb. 2007, pp. 1-4.

[11] S. Sergyan, "Color histogram features based image classification in content-based image retrieval systems," 2008 6th International Symposium on Applied Machine Intelligence and Informatics, Herlany, 2008, pp. 221-224

[12] T.-C. Lu and C.-C. Chang, "Color image retrieval technique based on color features and image bitmap," Inf. Process. Manage., vol. 43, no. 2, pp. 461-472, Mar. 2007.

[13] P. Poursistani, H. Nezamabadi-Pour, R. A. Moghadam, and M. Saeed, "Image indexing and retrieval in JPEG compressed domain based on vector quantization," Math. Comput. Model., vol. 57, nos. 5-6, pp. 1005-1017, 2013.

[14] J. M. Guo, H. Prasetyo and J. H. Chen, "Content-Based Image Retrieval Using Error Diffusion Block Truncation Coding Features," in IEEE Transactions on Circuits and Systems for Video Technology, vol. 25, no. 3, pp. 466-481, March 2015.

[15] T. Esther Ratna, Dr. N. Subash Chandra, "Color Features based Image Retrieval Framework: A Review”, IJCMS, Volume 7, Issue 2, February 2018

[16] Dr.M.Ashok and Dr. T. Bhaskar Reddy,"Color image compression based on Luminance and Chrominance using Binary Wavelet Transform (BWT) and Binary Plane Technique (BPT)". International Journal of Computer Science and Information Technology \& Security (IJCSITS), 1(2): 2249-9555-2012

[17] P.Ashok Babu, Dr. K.V.S.R. Prasad, "A lossy color image compression using IWT and BPT”, Graphics \& Vision, 12(15), 2012
https://sites.google.com/site/dctresearch/Home/content-basedimage-retrieval

ElAlami ME, "A novel image retrieval model based on the most relevant features", Knowledge-Based System 2011;24(1):23-32

[20] Ashraf R, Bashir K, Irtaza A, Mahmood MT, "Content based image retrieval using embedded neural networks with bandletized regions Entropy", 2015-17 (6):3552-80.

[21] Mutasem K. Alsmadi, "An efficient similarity measure for content based image retrieval using memetic algorithm", Egyptian Journal of Basic and Applied Sciences, 4 (2017) 112-122.

[22] G. Ramprabu, S. Nagarajan, "Design and Analysis of Novel Modified Cross Layer Controller for WMSN", Indian Journal of Science and Technology, Vol 8(5), March 2015, pp.438-444. 